\title{
Papel do óxido nítrico no desenvolvimento de lesões cardíacas na fase aguda da infecção experimental pelo Trypanosoma cruzi
}

\author{
Role of nitric oxide in the development of cardiac lesions during the \\ acute phase of experimental infection by Trypanosoma cruzi
}

\author{
Cláudia Renata Bibiano Borges ${ }^{1}$, Virmondes Rodrigues Junior ${ }^{1}$, Marlene Antônia dos Reis ${ }^{2}$, \\ Lúcio Roberto Castellano, Javier Emilio Lazo Chica ${ }^{3}$, Sanívia Aparecida de Lima Pereira ${ }^{4}$, \\ Edjane Souza Santos ${ }^{1}$ e Denise Bertulucci Rocha Rodrigues ${ }^{1,4}$
}

\begin{abstract}
RESUMO
A doença de Chagas é causada pelo Trypanosoma cruzi e o coração é o órgão mais acometido. 0 óxido nítrico apresenta importante ação antiTrypanosoma, porém, com pouca evidência de seu papel no mecanismo de lesão tecidual. 0 objetivo deste estudo foi analisar a contribuição do óxido nítrico no desenvolvimento da inflamação e da fibrose cardíaca na fase aguda da infecção experimental por cepas Y e Colombiana do Trypanosoma cruzi. A inflamação foi significativamente maior nos animais infectados pela cepa Colombiana, comparada com os infectados com a cepa Y, tanto nos animais C57BL/6 (3,98x1,87\%; $\mathrm{p}=0,004)$ quanto nos animais C57BL/6 deficientes na sintase do óxido nítrico induzível (3,99x2,4\%; $\mathrm{p}=0,013)$. 0 parasitismo cardíaco dos animais C57BL/6 deficientes na sintase do óxido nítrico induzível infectados pela cepa Colombiana foi significativamente maior que o destes mesmos animais infectados com a cepa $\mathrm{Y}\left(2,78 \mathrm{x} 0,17\right.$ ninhos $\left./ \mathrm{mm}^{2} ; \mathrm{p}=0,004\right)$ assim como, os animais $\mathrm{C} 57 \mathrm{BL} / 6 \mathrm{infectados} \mathrm{com} \mathrm{a}$ cepa Colombiana $\left(2,78 \mathrm{x} 1,33\right.$ ninhos $\left./ \mathrm{mm}^{2} ; \mathrm{p}=0,006\right)$ ou cepa $\mathrm{Y}\left(2,78 \mathrm{x} 0,53\right.$ ninhos $\left./ \mathrm{mm}^{2} ; \mathrm{p}=0,005\right)$. Os dados reforçam o papel do óxido nítrico no controle do parasitismo e sugerem seu papel na proteção tecidual, controlando a inflamação e potencialmente diminuindo lesões cardíacas durante a fase aguda na doença de Chagas experimental.
\end{abstract}

Palavras-chaves: Doença de Chagas. Óxido nítrico. Inflamação. Fibrose.

\section{ABSTRACT}

Chagas disease is caused by Trypanosoma cruzi and the heart is the organ most affected. Nitric oxide has notable anti-Trypanosoma action, but with little evidence regarding its role in the mechanism for tissue injury. The objective of this study was to analyze the contribution of nitric oxide towards the development of inflammation and cardiac fibrosis during the acute phase of experimental infection by $\mathrm{Y}$ and Colombian strains of Trypanosoma cruzi. The inflammation was significantly more intense in animals infected with the Colombian strain, compared with those infected with the $Y$ strain, both in C57BL/6 animals (3.98 vs 1.87\%; $\mathrm{p}=0.004)$ and in $\mathrm{C} 57 \mathrm{BL} / 6$ animals deficient in inducible nitric oxide synthase (3.99 vs $2.4 \% ; \mathrm{p}=0.013)$. The cardiac parasite load in inducible nitric oxide synthase-deficient C57BL/6 animals infected with the Colombian strain was significantly greater than in those infected with the $\mathrm{Y}$ strain $\left(2.78\right.$ vs. 0.17 nests $\left./ \mathrm{mm}^{2} ; \mathrm{p}=0.004\right)$, and also significantly greater than in the $\mathrm{C} 57 \mathrm{BL} / 6$ infected with both the Colombian strain (2.78 vs 1.33 nests $\left./ \mathrm{mm}^{2} ; \mathrm{p}=0.006\right)$ and $\mathrm{Y}$ strains $\left(2.78\right.$ vs 0.53 nests $\left./ \mathrm{mm}^{2} ; \mathrm{p}=0.005\right)$. The data confirm that nitric oxide has a role in parasite load control and suggest that it has a role in tissue protection, through controlling inflammation and potentially reducing cardiac lesions during the acute phase of Chagas disease.

Key-words: Chagas disease. Nitric oxide. Inflammation. Fibrosis.

\footnotetext{
1. Laboratório de Imunologia, Universidade Federal do Triângulo Mineiro, Uberaba, MG. 2. Disciplina de Patologia Geral, Universidade Federal do Triângulo Mineiro, Uberaba, MG. 3. Disciplina de Biologia Celular, Universidade Federal do Triângulo Mineiro, Uberaba, MG. 4. Universidade de Uberaba, Uberaba, MG. Apoio Financeiro: FAPEMIG, CNPq, CAPES, FUNEPU.

Endereço para correspondência: Profa. Denise Bertulucci Rocha Rodrigues. Laboratório de Imunologia/UFTM. Av. Getúlio Guaritá s/n, 38025-180 Uberaba, MG. Tel: 5534 3318-5289

e-mail: denise.rodrigues@uniube.br

Recebido para publicação em 01/12/2008

Aceito em 20/03/2009
}

A doença de Chagas possui caráter endêmico em largas extensões territoriais do continente americano e apesar dos recentes progressos no controle da doença, aproximadamente 20 milhões de pessoas ainda estão cronicamente infectadas ${ }^{12}$. A fase aguda, que pode ser ou não sintomática ${ }^{25}$, é seguida por um longo período de latência. 0 grau de comprometimento das células cardíacas varia desde formas discretas, assintomáticas até formas graves, eventualmente fatais ${ }^{18}$. Estudos demonstram que o infiltrado inflamatório parece ser mais lesivo para as fibras 
cardíacas do que para os próprios parasitas ${ }^{2033}$. A patogênese da miocardite chagásica está diretamente relacionada com a presença do parasito e ao padrão de resposta imune do hospedeiro ${ }^{15} 172224$. Na resposta inflamatória, o papel das citocinas na susceptibilidade e resistência à infecção pelo Trypanosoma cruzi ainda não foi totalmente elucidado. 0 interferon gama (IFN- $\gamma$ ), é considerado uma citocina protetora, especialmente na fase aguda pela capacidade de ativar a expressão da sintase do óxido nítrico induzível ${ }^{72632}$. Sozinho ou associado ao fator de necrose tumoral alfa (TNF- $\alpha$ ), o IFN- $\gamma$ é um dos melhores indutores da atividade microbicida de macrófagos em várias infecções por parasitas intracelulares, incluindo Trypanosoma cruzi $i^{2328} 290$. 0 óxido nítrico é uma importante molécula microbicida, sendo que camundongos deficientes em receptor de IFN- $\gamma\left(\right.$ IFN- $\left.\gamma \mathrm{R}^{-/}\right)$ou deficientes na enzima sintase de óxido nítrico induzível apresentaram elevada susceptibilidade a infecção por Trypanosoma cruzi, com aumento da parasitemia e mortalidade durante a fase aguda $\mathrm{a}^{16}$. Camundongos $\mathrm{NOS}^{--}$apresentam dilatação ventricular progressiva e na disfunção sistólica durante miocardite aguda ocasionada pela cepa Tulahuen de Trypanosoma cruzi $^{10}$. Por outro lado, estes mesmos animais apresentam menor desnervação do plexo mesentérico do esôfago após a infecção pela cepa $Y^{5}$. 0 óxido nítrico apresenta ainda potencial imunoregulatório e antifibrótico verificado na infecção experimental pelo Schistosoma mansoni $i^{14}$. Devido às suas diferentes propriedades, o óxido nítrico pode ter um papel importante no controle do parasita como também no desenvolvimento das lesões, assim neste trabalho analisamos a contribuição do óxido nítrico no controle do parasitismo, no desenvolvimento da inflamação e da fibrose cardíaca na fase aguda da infecção experimental por duas diferentes cepas do Trypanosoma cruzi.

\section{MATERIAL E MÉTODOS}

Animais e infecção. Foram analisados 24 camundongos, separados em 4 grupos de 6 animais: Grupo 1 (G1): linhagem C57BL/6 infectado com a cepa Y; Grupo 2 (G2): C57BL/6 infectado com a cepa Colombiana; Grupo 3 (G3): $\mathrm{C} 57 \mathrm{BL} / 6 \mathrm{iNOS}^{-/}$infectado com a cepa $Y$ e Grupo 4 (G4): C57BL/6 iNOS $^{-1}$ infectado com a cepa Colombiana. Os animais foram infectados com 3.000 formas tripomastigotas da cepa $\mathrm{Y}$ ou da cepa Colombiana do Trypanosoma cruzi por via intraperitoneal. A eutanásia foi realizada no $15^{\circ}$ dia após a inoculação, através do deslocamento cervical e posterior autópsia, com incisão ventral, para a retirada do coração. Os animais foram mantidos e eutanasiados seguindo as recomendações do Colégio Brasileiro de Experimentação Animal (COBEA) ${ }^{1221}$.

Processamento das lâminas. 0s fragmentos do coração foram processados e as lâminas confeccionadas e submetidas às colorações por HE para quantificação do infiltrado inflamatório, por picrosírius para avaliação morfométrica do tecido conjuntivo fibroso. Ainda, lâminas foram utilizadas na técnica de imunohistoquímica para detecção dos ninhos de Trypanosoma cruzi, utilizando soro de coelho antiTrypanosoma cruzi.

Quantificação do infiltrado inflamatório e densidade parasitária. Para quantificação do infiltrado inflamatório, as imagens foram capturadas, analisadas no programa Image J
(NIH, BETHESDA, USA) e calibradas através da lâmina (Leica) com régua graduada em dois milímetros divididos em unidades de $0,01 \mathrm{~mm}$ (dez micrômetros) na objetiva de 10x. Foi utilizado um sistema teste de contagem de pontos em uma área teste prédeterminada ${ }^{19}$. Para calcular o número de áreas teste ou campos, foi utilizada a fórmula de Hally ${ }^{13}$. Para análise da miocardite, empregou-se o critério de Dallas ${ }^{6}$.

A densidade parasitária do número de ninhos de amastigotas por $\mathrm{mm}^{2}$ foi realizada através da contagem desses ninhos em todo o corte do coração de cada animal. A quantificação da área do miocárdio foi realizada capturando toda a imagem do coração com o auxílio de um scanner HP Scanjet G 4050 (USA) e o programa Adobe Photoshop CS2. 0 tamanho de cada imagem corresponde a $10 \mathrm{~mm}^{2}$. As imagens foram analisadas usando o software Image J, e os valores expressos em ninhos $/ \mathrm{mm}^{2}$.

Quantificação da fibrose. A morfometria foi feita utilizando o Sistema Analisador de Imagem Automático KS 300 (Carl Zeiss, Alemanha). 0 campo a ser quantificado foi capturado por meio de uma câmera acoplada ao microscópio e ao computador para digitalização da imagem. Na imagem polarizada o tecido conjuntivo fibroso apresentou-se birrefringente sendo marcado pelo observador, obtendo assim o percentual de fibrose por área analisada. A análise foi realizada em todos os campos do fragmento, totalizando 20 campos $^{8}$.

Detecção de ninhos de Trypanosoma cruzi. A imunohistoquímica para detecção de ninhos de Trypanosoma cruzi foi realizada nos cortes de coração fixados em formol. Após bloqueio da peroxidase endógena as lâminas foram incubadas com 0 anticorpo antiTrypanosoma cruzi produzido em coelho (1:250), durante 2 horas à temperatura ambiente $\left(37^{\circ} \mathrm{C}\right)$. Posteriormente, as lâminas foram incubadas com a proteína A conjugada com peroxidase (1:500) e reveladas com $\mathrm{H}_{2} \mathrm{O}_{2} .0,05 \%$ + DAB-diaminobenzidina, em tampão tris-HCl pH 7,4. Os cortes foram contracorados com hematoxilina e as lâminas foram então montadas para análise em microscópio de luz comum8.

Análise estatística. A análise estatística foi realizada através do programa Statview (Abaccus, EUA). As variáveis apresentaram distribuição não normal e foram analisadas pelo teste de MannWhitney (T) para comparação de dois grupos independentes. Para as correlações entre as variáveis estudadas foi utilizado o Coeficiente de Correlação de Spearman. Os resultados foram considerados significativos quando a probabilidade foi menor que $5 \%(\mathrm{p}<0,05)$.

\section{RESULTADOS}

0 processo inflamatório foi avaliado por morfometria utilizando um sistema teste de contagem de pontos em uma área teste pré-determinada, na fase aguda no miocárdio dos animais infectados com as cepas Y e Colombiana. Não houve diferença significativa entre os animais $\mathrm{C} 57 \mathrm{BL} / 6$ e C57BL/6 $\mathrm{iNOS}^{-1}$ quando infectados pela cepa $\mathrm{Y}(1,87$ e $2,4 \%$, respectivamente) ou Colombiana (3,98 e 3,99 \%, respectivamente). Entretanto, em ambas as linhagens, quando comparados os animais infectados com as cepas Y e Colombiana, no grupo $\mathrm{C} 57 \mathrm{BL} / 6 \mathrm{iNOS}^{-1-}$ a 
inflamação causada pela cepa Colombiana foi significativamente maior $(3,99$ e $2,4 \%$, respectivamente; $\mathrm{p}=0,013)$ assim como, no grupo C57BL/6 (3,98 e 1,87\%, respectivamente; $\mathrm{p}=0,004)$ (Figura 1). 0 número de ninhos de Trypanosoma cruzi no tecido cardíaco nos animais infectados com a cepa Colombiana foi significativamente maior nos animais $\mathrm{C} 57 \mathrm{BL} / 6 \mathrm{iNOS}^{-/}$que nos C57BL/6 selvagens (2,78 e 1,33 ninhos/ $/ \mathrm{mm}^{2}$, respectivamente; $\mathrm{p}=0,006$ ), enquanto nos animais infectados com a cepa $\mathrm{Y}$ não foi observado diferença significativa entre estes grupos (Figura 2).

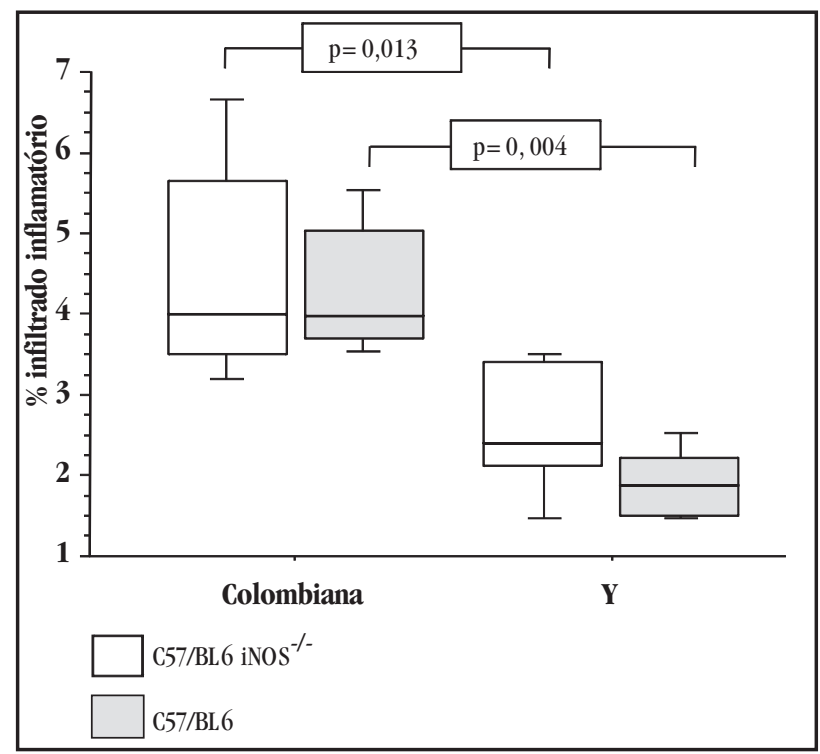

FIGURA 1

Distribuição da porcentagem de exsudato inflamatório em camundongos C57BL/6 e C57BL/6 iNOS $\%$ infectados pelas cepas Y e com a cepa Colombiana na fase aguda da infecção. A linha horizontal representa a mediana, a barra o percentil de $25 \%$ a $75 \%$ e a linha vertical representa o percentil de $10 \%$ a $90 \%$.

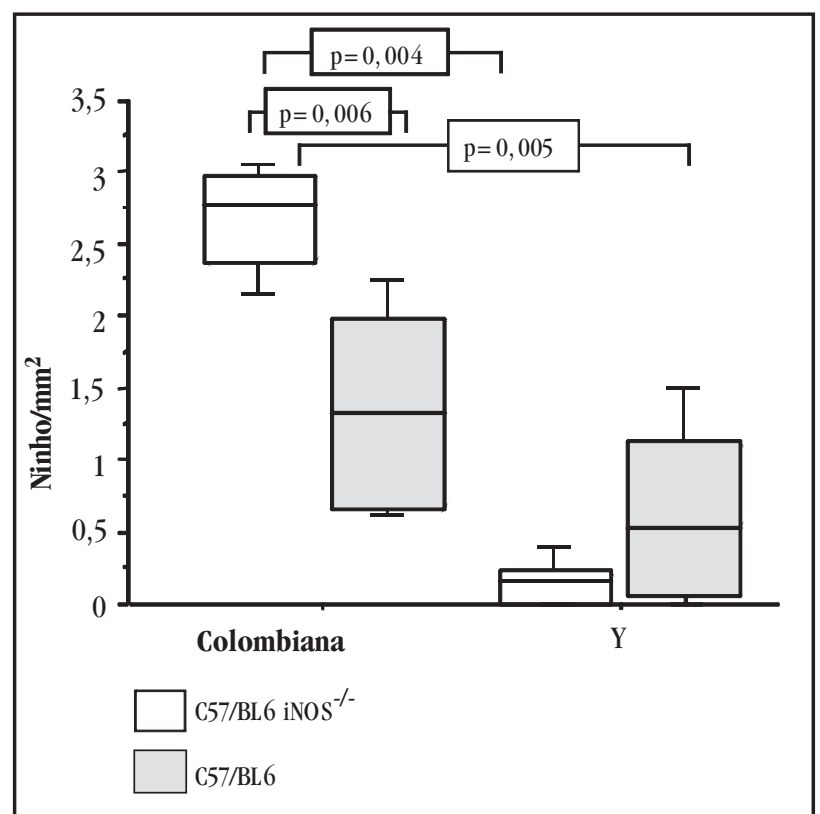

\section{FIGURA 2}

Distribuição do número de ninhos por $\mathrm{mm}^{2}$ em camundongos C57BL/6 e $\mathrm{C} 57 \mathrm{BL} / 6 \mathrm{iNOS}$ - infectados com a cepa Y e com a cepa colombiana na fase aguda. Os dados são apresentados em número de ninhos por área do corte analisado. A linha horizontal representa a mediana, a barra o percentil de $25 \%$ a $75 \%$, a linha vertical o percentil de $10 \%$ a $90 \%$.
Nos animais C57BL/6 $\mathrm{iNOS}^{-/}$infectados com a cepa Colombiana, o número de ninhos foi significativamente maior que nos animais C57BL/6 selvagens e C57BL/6 iNOS ${ }^{-/}$infectados com a cepa Y $(2,78$ e 0,53 ninhos $/ \mathrm{mm}^{2} ; \mathrm{p}=0,005$ e 2,78 e 0,17 ninhos $/ \mathrm{mm}^{2} ; \mathrm{p}=0,004$, respectivamente). A linhagem $\mathrm{C} 57 \mathrm{BL} / 6$ não houve diferença significativa quando comparada as duas cepas de Trypanosoma cruzi (Figura 2). Ainda, observamos uma correlação positiva e significativa quando comparamos em ambas as cepas e linhagens, a porcentagem de infiltrado inflamatório com o número de ninhos de amastigotas/ $\mathrm{mm}^{2}(\mathrm{rS}=0,002)$ (Figura 3). No entanto, embora a correlação entre a porcentagem de infiltrado inflamatório e de fibrose $/ \mathrm{mm}^{2}$ também tenha sido positiva, essa diferença não foi significativa (Figura 4). Também não houve diferença significativa na intensidade da fibrose entre os grupos estudados (Figura 5).

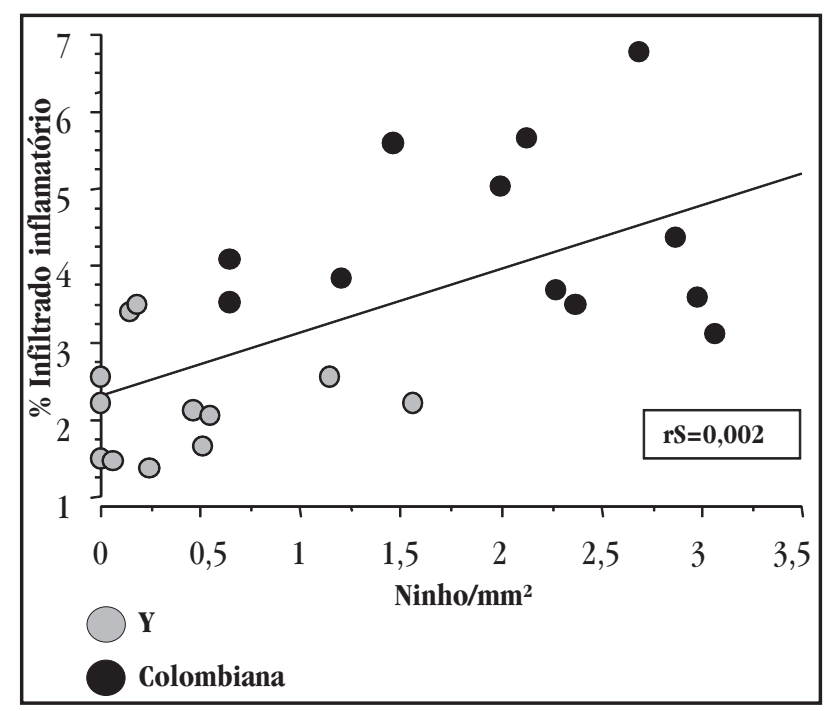

FIGURA 3

Correlação entre o número de ninhos por $\mathrm{mm}^{2}$ e a \% de infiltrado inflamatório no tecido cardíaco dos camundongos C57BL/6 e C57BL/6 iNOS \% infectados com as cepas Y e com a cepa Colombiana.

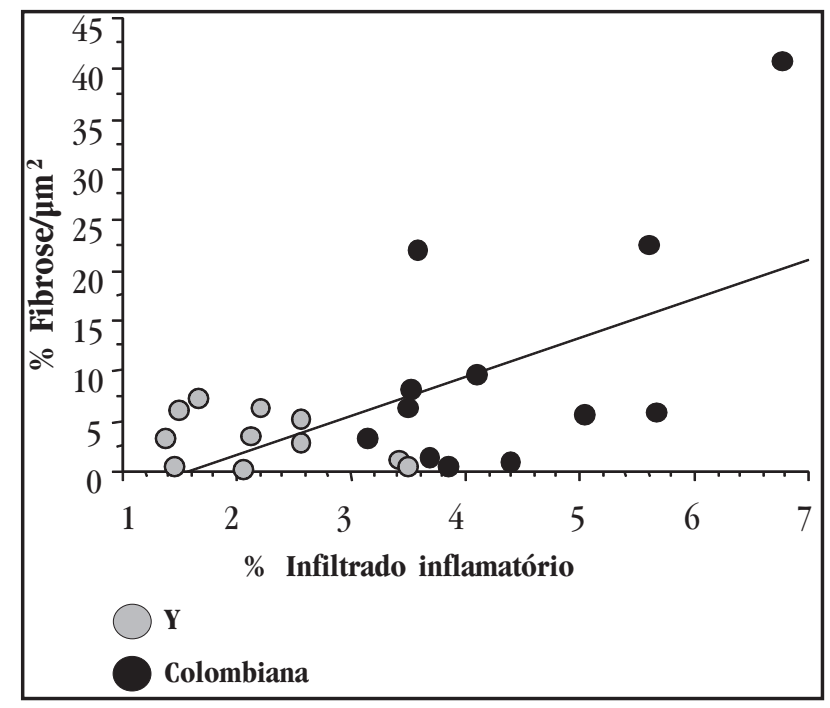

FIGURA 4

Correlação entre a \% de fibrose/ $\mathrm{\mu m}^{2}$ e a \% de infiltrado inflamatório no tecido cardíaco dos camundongos C57BL/6 e C57BL/6 iNOS \% infectados com as cepas Y e com a Cepa Colombiana. 


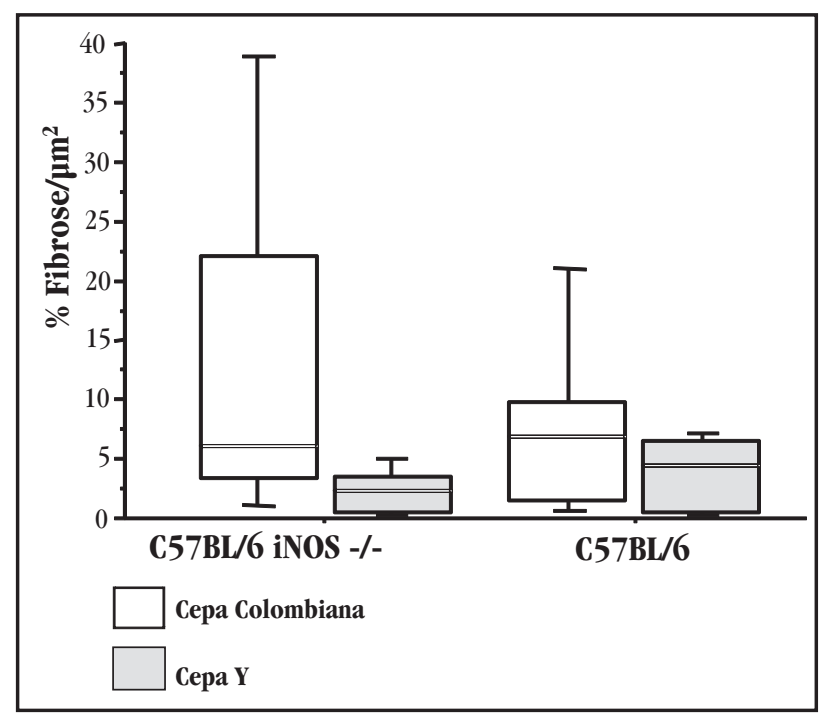

FIGURA 5

Distribuição da fibrose em camundongos C57BL/6 eC57BL/6 iNOS $\%$, infectados com a cepa Y e com a cepa Colombiana, durante a fase aguda da infecção. Os dados são apresentados em porcentagem da área de fibrose. A linha horizontal representa a mediana, a barra 0 percentil de $25 \%$ a $75 \%$, a linha vertical o percentil de $10 \%$ a $90 \%$.

\section{DISCUSSÃO}

Nossos resultados apontam que o número de ninhos de Trypanosoma cruzi no tecido cardíaco nos animais infectados com a cepa Colombiana foi significativamente maior nos animais C57BL/6 iNOS $־$ que nos animais C57BL/6 devido a sua deficiência de produção de óxido nítrico, uma importante molécula tripanocida ${ }^{1130}$. Comparando os animais $\mathrm{C} 57 \mathrm{BL} / 6 \mathrm{iNOS}^{-/} \mathrm{com}$ as duas cepas, os animais infectados com a cepa Colombiana, apresentaram números de ninhos significativamente maiores que os infectados com a cepa Y. Esses resultados se justificam, uma vez que a cepa Colombiana apresenta nítido miotropismo, sobretudo com envolvimento de musculatura esqueléticát. Já a cepa Y é caracterizada por uma infecção de evolução rápida, e com altos níveis de parasitemia e apresenta tropismo por macrófagos ${ }^{3}$. Os animais da linhagem C57BL/6, em nossos resultados, apresentaram um padrão de resistência à infecção pelo Trypanosoma cruzi em relação aos C57BL/ $6 \mathrm{iNOS}^{-1}$. Animais C57BL/6 têm sido apontados como resistentes a parasitas intracelulares, como Trypanosoma cruzi e Leishmania $s p$ apresentando capacidade de gerar uma resposta imune predominante de padrão Th1 capaz de estimular 0 macrófago a eliminar parasitas intracelulares ${ }^{27}$.

No presente trabalho, comparando a inflamação durante a fase aguda entre os grupos de animais C57BL/6 e C57BL/6 iNOS $^{-/}$infectados com a cepa Colombiana e com a cepa Y não houve diferença significativa. No entanto, em cada uma das linhagens, quando comparados os animais infectados com as duas cepas, no grupo C57BL/6 iNOS $^{-/}$a inflamação causada pela cepa Colombiana foi significativamente maior que a causada pela cepa Y. Encontramos uma correlação positiva e significativa entre o aumento do infiltrado inflamatório e o número de parasitas encontrados nos tecidos analisados, muito provavelmente devido à cepa Colombiana apresentar miotropismo e induzir inflamação com maior intensidadé . Já quando analisamos a fibrose, observamos uma correlação positiva, mas não significativa entre essa variável e o infiltrado inflamatório. Neste estudo, quando comparados os animais $\mathrm{C} 57 \mathrm{BL} / 6$ e $\mathrm{C} 57 \mathrm{BL} / 6 \mathrm{iNOS}^{-1}$, nas duas cepas, não houve diferença significativa, embora fosse observado que os animais $\mathrm{C} 57 \mathrm{BL} / 6 \mathrm{iNOS}^{-}$apresentaram uma maior área de tecido conjuntivo fibroso quando infectados pela cepa Colombiana. Em modelo de fibrose induzida pela infecção experimental pelo Schistosoma mansoni, foi demonstrado que a sintase do óxido nítrico induzível é responsável pelos efeitos antiinflamatórios e anti-fibróticos atribuídos a uma resposta Th $1^{14}$. Animais deficientes em sintase do óxido nítrico induzível apresentaram maior inflamação, provavelmente devido ao maior parasitismo tissular, sugerindo que a inflamação da fase aguda é dependente e proporcional ao parasitismo tissular ${ }^{31}$. Não foi observada neste estudo, diferença na fibrose entre os animais deficientes e sintase do óxido nítrico induzível e seu homólogos selvagens sugerindo que na doença de Chagas, o óxido nítrico não está envolvido na formação precoce da fibrose cardíaca.

\section{REFERÊNCIAS}

1. Abel LC, Rizzo LV, Ianni B, Albuquerque F, Bacal F, Carrara D, Bocchi EA, Teixeira HC, Mady C, Kalil J, Cunha-Neto E. Chronic Chagas' disease cardiomyopathy patients display an increased IFN-gamma response to Trypanosoma cruzi infection. Journal of Autoimmunity 17:99-107, 2001.

2. Almeida-Leite CM, Galvão LM, Afonso LC, Cunha FQ, Arantes RM. Interferongamma induced nitric oxide mediates in vitro neuronal damage by Trypanosoma cruzi-infected macrophages. Neurobiology of Disease 25:170-178, 2007.

3. Andrade S. Caracterização de cepas do Trypanosoma cruzi isoladas no Recôncavo Baiano. Revista de Patologia Tropical 3:65-121, 1974.

4. Andrade Z. Patologia da doença de Chagas. In: Brener Z, Andrade Z, Barral-Neto M (eds) Trypanossoma cruzi e Doença de Chagas. Guanabara Koogan, Rio de Janeiro, p.201-230, 2000.

5. Arantes RM, Marche HH, Bahia MT, Cunha FQ, Rossi MA, Silva JS. Interferongamma-induced nitric oxide causes intrinsic intestinal denervation in Trypanosoma cruzi-infected mice. American Journal of Pathology 164:13611368, 2004.

6. Aretz HT. Myocarditis: the Dallas criteria. Human Pathology 18:619-624, 1987.

7. Bahia-Oliveira LM, Gomes JA, Cançado JR, Ferrari TC, Lemos EM, Luz ZM, Moreira MC, Gazzinelli G, Correa-Oliveira R. Immunological and clinical evaluation of chagasic patients subjected to chemotherapy during the acute phase of Trypanosoma cruzi infection 14-30 years ago. The Journal of Infectious Diseases 182:634-638, 2000.

8. Caliari MV, de Lana M, Caja RA, Carneiro CM, Bahia MT, Santos CA, Magalhães GA, Sampaio IB, Tafuri WL. Immunohistochemical studies in acute and chronic canine chagasic cardiomyopathy. Virchows Archiv 441:69-76, 2002.

9. Chagas C. Processos patojenicos da tripanozomiase americana. Memórias do Instituto Oswaldo Cruz 8: 5-35, 1916.

10. Chandra M, Tanowitz HB, Petkova SB, Huang H, Weiss LM, Wittner M. Factor SM, Shtutin V, Jelicks LA, Chan J, Shirani J. Significance of inducible nitric oxide synthase in acute myocarditis caused by Trypanosoma cruzi (Tulahuen strain). International Journal for Parasitology 32:897-905, 2002.

11. Gazzinelli RT, Oswald IP, Hieny S, James SL, Sher A. The microbicidal activity of interferon-gamma-treated macrophages against Trypanosoma cruzi involves an L-arginine-dependent, nitrogen oxide-mediated mechanism inhibitable by interleukin-10 and transforming growth factor-beta. European Journal of Immunology 22:2501-2506, 1992.

12. Guimarães M, Mázaro R. Princípios Éticos e Práticos do Uso de Animais de Experimentação. Universidade Federal de São Paulo, São Paulo, 2004. 
13. Hally A. A counting method for measuring the volumes of tissue components in microscopical sections. The Quarterly Journal of Microscopical Science 105:503, 1964.

14. Hesse M, Cheever AW, Jankovic D, Wynn TA. NOS-2 mediates the protective anti-inflammatory and antifibrotic effects of the Th1-inducing adjuvant, IL-12, in a Th2 model of granulomatous disease. The American Journal of Pathology 157:945-955, 2000

15. Higuchi ML. Chagas disease. Importance of the parasite in the pathogenesis of the cardiac chronic disease. Arquivos Brasileiros de Cardiologia 64:251-254, 1995.

16. Holscher C, Kohler G, Muller U, Mossmann H, Schaub GA, Brombacher F. Defective nitric oxide effector functions lead to extreme susceptibility of Trypanosoma cruzi-infected mice deficient in gamma interferon receptor or inducible nitric oxide synthase. Infection and Immunity 66:1208-1215, 1998

17. Jones EM, Colley DG, Tostes S, Lopes ER, Vnencak-Jones CL, McCurley TL. Amplification of a Trypanosoma cruzi DNA sequence from inflammatory lesions in human chagasic cardiomyopathy. The American Journal of Tropical Medicine and Hygiene 48:348-357, 1993

18. Lopes E, Chapadeiro E. Anatomia patológica da doença de Chagas humana. In: Dias J, Coura J (eds) Clínica e terapêutica da doença de Chagas. Uma abordagem prática para o clínico geral. Fundação Oswaldo Cruz, Rio de Janeiro, p.67-84, 1997.

19. Mandarim-de-Lacerda CA. Stereological tools in biomedical research. Anais da Academia Brasileira de Ciências 75:469-486, 2003.

20. Mayer B, Schmidt K, Humbert P, Bohme E. Biosynthesis of endothelium-derived relaxing factor: a cytosolic enzyme in porcine aortic endothelial cells Ca2+dependently converts L-arginine into an activator of soluble guanylyl cyclase. Biochemical and Biophysical Research Communications 164:678-685, 1989.

21. Mezadri T, Thomáz V, Amaral V. Animais de laboratório: 9 cuidados na iniciação experimental. Florianópolis, 2004

22. Mortara RA, Procopio DO, Barros HC, Verbisck NV, Andreoli WK, Silva RB, Silva S. Features of host cell invasion by different infective forms of Trypanosoma cruzi. Memórias do Instituto Oswaldo Cruz 94 (supl 1):135-137, 1999.

23. Munoz-Fernandez MA, Fernandez MA, Fresno M. Activation of human macrophages for the killing of intracellular Trypanosoma cruzi by TNF-alpha and IFN-gamma through a nitric oxide-dependent mechanism. Immunology Letters 33:35-40, 1992.

24. Palomino SA, Aiello VD, Higuchi ML. Systematic mapping of hearts from chronic chagasic patients: the association between the occurrence of histopathological lesions and Trypanosoma cruzi antigens. Annals of Tropical Medicine and Parasitology 94:571-579, 2000.

25. Rassi A. Clínica: fase aguda. In: Brener Z, Andrade Z (eds) Trypanosoma cruzi e doença de Chagas. Guanabara Koogan, Rio de Janeiro, p.249-264, 1979.

26. Reed SG. In vivo administration of recombinant IFN-gamma induces macrophage activation, and prevents acute disease, immune suppression, and death in experimental Trypanosoma cruzi infections. The Journal of Immunology 140:4342-4347, 1988

27. Sacks D, Noben-Trauth N. The immunology of susceptibility and resistance to Leishmania major in mice. Nature Reviews Immunology 2:845-858, 2002

28. Sher A, Coffman RL. Regulation of immunity to parasites by T cells and T cellderived cytokines. Annual Review of Immunology 10:385-409, 1992.

29. Silva JS, Morrissey PJ, Grabstein KH, Mohler KM, Anderson D, Reed SG. Interleukin 10 and interferon gamma regulation of experimental Trypanosoma cruzi infection. The Journal of Experimental Medicine 175:169-174, 1992.

30. Silva JS, Vespa GN, Cardoso MA, Aliberti JC, Cunha FQ. Tumor necrosis factor alpha mediates resistance to Trypanosoma cruzi infection in mice by inducing nitric oxide production in infected gamma interferon-activated macrophages. Infection and Immunity 63:4862-4867, 1995

31. Toledo MJ, Bahia MT, Veloso VM, Carneiro CM, Machado-Coelho GL, Alves CF, Martins HR, Cruz RE, Tafuri WL, Lana M. Effects of specific treatment on parasitological and histopathological parameters in mice infected with different Trypanosoma cruzi clonal genotypes. The Journal of Antimicrobial Chemotherapy 53:1045-1053, 2004.

32. Vespa GN, Cunha FQ, Silva JS. Nitric oxide is involved in control of Trypanosoma cruzi-induced parasitemia and directly kills the parasite in vitro. Infection and Immunity 62:5177-5182, 1994

33. Vianna G. Contribuição para o estudo da anatomia patolojica da "Molestia de Carlos Chagas": esquizotripanoze humana ou tireoidite parazitaria. Memórias do Instituto Oswaldo Cruz 3: 276-292, 1911. 